\title{
Model for the induction of spike timing- dependent plasticity by pre- and postsynaptic spike trains
}

\author{
Kristofor D Carlson ${ }^{*}$, Nicholas Giordano \\ From Nineteenth Annual Computational Neuroscience Meeting: CNS*2010 \\ San Antonio, TX, USA. 24-30 July 2010
}

Spike timing-dependent plasticity (STDP), a process in which changes in synaptic strength are determined by the relative timing of pre- and postsynaptic activity, has been studied and modeled by a number of researchers, but many questions still remain. It has been suggested that STDP involves a postsynaptic chemical network with stable states corresponding to long term potentiation (LTP) and long term depression (LTD). It is believed that the switching between these states is driven by the postsynaptic $\mathrm{Ca} 2+$ concentration, but the manner in which the $\mathrm{Ca} 2+$ dynamics is able to trigger either LTP or LTD, depending on the relative timing of pre- and postsynaptic activity remains unclear.

We have investigated a model of STDP that combines (1) the tristable chemical network involving CaMKII and PP2A studied by Pi and Lisman [1], with (2) compartmental modeling of backpropagating action potentials (BPAPs), N-methyl D-aspartate receptors (NMDARs), and voltage-dependent calcium channels (VDCCs). In previous work we have studied how this model responds when a presynaptic pulse arrives either shortly before or shortly after a postsynaptic pulse (a BPAP), and shown how this model leads naturally to LTP when the presynaptic pulse arrives first, or LTD when the postsynaptic pulse arrives first, in agreement as found in experimental studies (e.g., [2] and [3]). The response to spike triplets and other more complex preand postsynaptic spike trains are also of interest. Experiments [4] have shown that the response to such multispike trains is not simply the sum of the responses to the component spike pairs. For example, the response to a spike triplet consisting of pre-post-presynaptic spikes is often not explained by the simple addition of the responses to a pre-post spike pair followed by a post-pre spike pair. Previous work has proposed only heuristic rules for such multispike responses. In this paper we describe the application of our model of STDP to multispike situations. Our model exhibits a non-additive response similar to that observed by Wang et al. [4], and gives insight into how this non-additivity arises from properties of the CaMKII/PP2A network.

Published: 20 July 2010

\section{References}

1. Pi HJ, Lisman JE: Coupled Phosphatase and Kinase Switches Produce the Tristability Required for Long-Term Potentiation and Long-Term Depression. J Neurosci 2008, 28:13132-13138.

2. Bi GQ, Poo MM: Synaptic Modifications in Cultured Hippocampal Neurons: Dependence on Spike Timing, Synaptic Strength, and Postsynaptic Cell Type. J Neurosci 1998, 18:10464-10472.

3. Markram H, Lubke J, Frotscher M, Sakmann B: Regulation of Synaptic Efficacy by Coincidence of Postsynaptic APs and EPSPs. Science 1997, 275:213-215.

4. Wang HX, Gerkin RC, Nauen DW, Bi GQ: Coactivation and TimingDependent Integration of Synaptic Potentiation and Depression. Nat Neurosci 2005, 8:187-193.

doi:10.1186/1471-2202-11-S1-P188

Cite this article as: Carlson and Giordano: Model for the induction of spike timing-dependent plasticity by pre- and postsynaptic spike trains. BMC Neuroscience 2010 11(Suppl 1):P188.

\footnotetext{
* Correspondence: carlsokd@purdue.edu

Department of Physics, Purdue University, West Lafayette, IN 47907-2036,

USA
} 